\title{
The changes on intimate partner violence against women in the health system in Portugal
}

\author{
Raquel Cardoso, José Ornelas \\ Instituto Superior de Psicologia Aplicada, Instituto Universitário, Lisbon, Portugal; raquelvieitascardoso@gmail.com
}

Received 22 April 2013; revised 23 May 2013; accepted 2 July 2013

Copyright (C) 2013 Raquel Cardoso, José Ornelas. This is an open access article distributed under the Creative Commons Attribution License, which permits unrestricted use, distribution, and reproduction in any medium, provided the original work is properly cited.

\begin{abstract}
In Portugal, over the last 20 years, we have seen great strides in addressing intimate partner violence against women by their male partners. Measuring and understanding progress, and achieving sustainable systems change is a major challenge and to achieve this aim we need to identify events, persons and settings that contribute for the critical factors that create long term transformations in the systems and the creation of new resources [1]. Since the healthcare system is one of the main actors of intervention with survivors, the goal of this project was to interview key stakeholders from the healthcare system in order to understand the main changes they perceive have happened over the past two decades. Based on interviews with key stakeholders, our study explores the evidences of the changes that occurred in the healthcare system, the intersections with other systems and their visions for the future.
\end{abstract}

Keywords: Social Change; Intimate Partner Violence against Women; Health System

\section{INTRODUCTION}

Intimate partner violence against women has been a social issue and a concern for societies across the world and a recognized widespread problem that causes devastating physical, psychological and economic damage to people [2].

The last 20 years have seen great strides in addressing intimate partner violence against women by their male partners, all over the World. Shepard [3], mentions "an industry of intimate partner violence programs has emerged in criminal justice, human service, and Healthcare systems that did not previously exist. Still, intimate partner violence persists in our communities as a serious social concern” (p. 436). With all this work came the recognition that it requires a response from a variety of community sectors [4] and the need to reflect on the way that communities have organized to face this issue provided by research (e.g.: Lehner, Allen, 2009; Allen, 2006; Allen, Bybee, Sullivan, 2004).

Measuring progress and achieving sustainable systems change is a major challenge requiring the implementation of new policies, programs and different structures to support desired outcomes [5] it is important to identify how events, persons and settings contribute for the critical factors that create long term transformations in the systems and the creation of new resources [1].

Over the last years, in Portugal, the exchange of different and interdisciplinary perspectives has contributed to the need of addressing this social problem [6]. In the last 20 years we have seen many changes in this area. An increase in the services available for survivors, changes in the laws, in the training for professional and the changes in institutional proceedings and a growing involvement of professionals and communities in addressing the needs for protection, safety and resources of survivors and to raise awareness.

Since violence can have such a serious health impact on women, ranging from physical injuries to depression and suicide [7], that the WHO has stated the need for it to be addressed in a more comprehensive and holistic manner applying a public health approach [8], it is fundamental to include analysis of the changes occurring in the health system.

Many women seeking healthcare services are victims of intimate partner violence [9]. Examination of systems changes is an important goal of research on intimate partner violence against women because it presents an opportunity to understand the effects of interventions that aim to change in the systems, namely to understand the functions and patterns that are required in the response to violence and the leverages changes in those systems that improve the response to survivors [10]. 
"Social Change is not a consequence of discrete manmade interventions, but rather the change that occurs as a result of the evolution of the functions in a society. $(\cdots)$ the change agent is neither a passive observer, nor the final architect for plans, but a creative participant working with communities in the design and reorganization of their activities.” (p. 83) [11].

To create sweeping and transformative impact on communities and service delivery systems, shift the underlying infrastructure within a community or target context to support a desired outcome, including shifting existing policies and practices, resource allocation, relational structures, community norms and values, and skills and attitudes [12].

It is important to identify how and why interventions are effective but also to understand under what circumstances they provide desired effects, to identify the critical ingredients of change [1].

We argue on the need to understand the changes in the health system related to intimate partner violence against women in the complexities of both the system, the social problem and the different stakeholders involved. Change efforts are targeted at systems of vastly different scale and complexities, from those focused on individual programs, units within organizations to networks of organizations to whole communities [13]. We also understand that efforts to reform the healthcare systems response to intimate partner violence have been difficult.

Identifying the main factors that facilitated the changes in the health system over the past two decades is central to understanding how to pursue a more effective intervention regarding intimate partner violence against women.

\section{CURRENT STUDY}

The massive changes in policies, funding and services in intimate partner violence, are an example of a social change movement to create long term widespread transformation, through institutional reform and/or fundamental cultural changes [14]. The changes in how social institutions respond to survivors reflect a shift in cultural sanctions for violence against women and have a ripple effect in the daily lives of women [3].

The current study engages in an examination of how the health system has evolved over the past twenty years in what relates to intimate partner violence. To understand the progresses that took place in this field, we began by learning the national framework of the health system in Portugal, namely trough the general law applicable, and collecting documental evidence of health programs and policies regarding intimate partner violence protocols, the awareness raising strategies, training policies and funding. In a second step we interviewed key stakeholders that, due to their professional activities had a significant role designing and implementing those changes. We expect that the participants will be able to provide an inside overview of the main transformations that have occurred in the Portuguese health system that contribute to a more effective service providing and insights for future developments.

\section{METHODS}

\subsection{Sample}

We wanted to include participants that due to their job assignment and involvement with intimate partner violence issues could be key informants of the processes that occurred in the health system over the past twenty years. They should be from different professional backgrounds and from different parts of the country. It was important that those key informants could have had influence in promoting the changes. Five female participants were interviewed, a retired medical doctor that has coordinated the maternal health regional department for several years, a psychologist from a rural area that worked in the local network for intimate partner violence issues, the director of the regional forensics office, a medical doctor who has done research on intimate partner violence and pregnancy, a nurse that represents the regional health department for the issues of intimate partner violence.

\subsection{Procedures and Measures}

Regarding the collection of documental evidences that support the changes in the health system in Portugal, we analyzed the four National Action Plans on Domestic Violence, focusing on specific measures targeting the healthcare providers and facilities, specific programs and awareness raising campaigns. We also collected the various legislation concerning intimate partner violence and the sources of funding for health projects. This process gave us an overview of the main activities and persons engaged in promoting actions to change.

A semi structured protocol was developed by the members of the research group on intimate partner violence against women, to gather the participants and to generate discussion on the changes that have occurred in the health system and how those changes are having impacts on current policies and on future outcomes. The protocol was also reviewed by experts working in the services providing area. All selected participants had a vast experience in the field and brought a rich perspective of the influences that the health system has suffered.

The participants were selected from a group of well known professionals working in the health system and invited to participate in the study. They were interviewed in person using a semi structured protocol at a location of their choice. One of the participants answered in writing 
due to lack of availability. Interviews were audiotaped with permission and transcribed verbatim.

\subsection{Data Analysis}

All the documental evidences where organized by date and content in order to provide systematic information on the policies and measures for intimate partner violence in the health system.

All transcripts where reviewed by the first author using a content analysis methodology [15]. A first reading provided us with an overview of the contents and the main issues approach by the participants as pre-analyses notes and coding of the most important information. Finally, six categories were created that would provide insights on the evolution of the health system, starting with the preconditions that where needed for the issue to be approached; what are the understandings on intimate partner violence against women that shaped the participants approach and priorities, because it is important how the problems are identified, contested and labelled [16]; changes they have observed and implemented over the past twenty years; the impacts of those changes in the health system, paying a special attention to negative and positive in the health system in healthcare providers and consumers; the social impacts and awareness raising the participants perceived; and what are the changes needed and the challenges for the future. A final reading of the interviews was made to understand the intersections of those changes across other systems, namely, how the health system was influenced by the other systems that are involved in the intervention with survivors of intimate partner violence against women.

\section{RESULTS}

\subsection{Documental Evidences}

With the documental evidences that we were able to retrieve we can see that over the years, several legislation, measures and programs have been used to influence change in the health system. Some of this documents area interconnected with the healthcare system approach to intimate partner violence. The first legislation that punished domestic abused was passed in 1988. In 1991, a new legislation was passed, and it required that the victim would have to press charges. In 2000, the crime was turned public and any person who had knowledge of a situation had a mandatory obligation to report it. In 2009 a new law on prevention of intimate partner violence and victims' rights was passed, requiring services to provide access to healthcare, preventing secondary victimization, requiring victims to be assisted by specialized healthcare professional, and free of charge. It required also that those healthcare professional would be provided with specific training for early detection, and care. Awareness raising materials would be provided to be displayed in healthcare facilities. Since the year 2000, Portugal has had four, tree-year long, National Action Plans on Domestic Violence and some of the measures planned for the healthcare system were included in these Plans. In the years 2000-2006, a European Health Program provided resources for training and other materials in the health system. Research on health and intimate partner violence also took place. For instance, in 2006-2007, the Health Ministry funded a study to estimate the additional health care costs to the Portuguese National Health Service of intimate partner violence. The results suggest an additional health care cost of $140 €$ per year per victim of domestic violence, that is about $22 \%$ higher than health care costs of non-victims. A large proportion (90\%) of the additional costs associated with domestic violence is supported by the NHS, where consultations and drugs are the most important contributors of such costs. Health consequences of domestic violence result from losses in quality of life and worst health status of victims and correspond to additional permanent economic costs [17].

\subsection{Interviews}

Regarding our participants we can clearly state that they all have had professional positions in the health system that allows them to present a clear picture of the changes that happened since the early 1990s. Most of them have several years of professional experiences in decision making and only one has recently taken office in the field of intimate partner violence against women. Based on their insights we will be presenting the main finding in our interviews.

\subsubsection{Background Preconditions for Changes}

To understand how the participants view the changes in the Portuguese society that contributed for change on intimate partner violence they were invited to comment on how they came to work in this issue. The participants referred some experiences in their professional career that strongly contributed to their involvement. They also refer changes that have occurred on the status of women, personal experiences dealing with the consequences of intimate partner violence and opportunities inside the system that gave way to new areas of intervention.

"Before the Revolution in 1974, family planning was forbidden; women would come to the hospital and would state-my husband will not allow me" (P4). "Medicine has always worked with violence, most of the time with the victims, $(\cdots)$ but not like regular violence. $(\cdots)$ A case has marked us; a woman we had seen a few days earlier, complaining of intimate partner violence had been killed" (P5) "The changes in the Health Ministry, and the 
organizational changes where I work made me realize that there were many victims of domestic abuse, requiring services provision" (P2).

\subsubsection{Understandings about Intimate Partner Violence against Women}

Our participants have different understandings on intimate partner violence against women. They all use different approaches. There is no consensus on what is the framework. From the public health and Human Rights approach to the legal definition or a cultural problem, some of the participants state that it is not a women's problem, it also affects man, children, seniors or people with disabilities. One of the participants refers that "the main causes of intimate partner violence are mental health problems, alcohol and substance abuse and crisis situations of the abuser" (P1). Another participant analyses the need to approach violence from different perspectives "It must be seen in different perspectives, psychological, community, social, legal and medical." (P2). Yet another participants says it is a "gender violence problem" (P4), another "a problem of violence between family members" (P5).

\subsubsection{Changes over the Past Twenty Years}

The most important change of our participants' outline is the change in the national legislation. The changes in the Law in 2000, made it possible for anyone to report the crime to the police. Thought this doesn't happen most of the times, and survivors are the ones pressing charges, it facilitated the intervention of healthcare professionals. These changes also created new opportunities for the development of new working units dealing with domestic abuse and intimate partner violence, in the health system.

"The most important change was the legislation" (P1), (P2) (P3) (P4) (P5).

According to our participants, another instrument that proved to be very useful to foster the changes in the healthcare system was the four National Action Plans on Domestic Violence. Those plans contemplated several measures to be implemented in most systems working with violence, namely, the role of different professionals, funding and public awareness campaigns.

"Campaigns were made and films were on display in all healthcare facilities" (P4).

The changes also benefited from the investment on training. "Many healthcare professionals had the chance to have specific training on domestic abuse and intimate partner violence, provided in partnership with community organizations, to improve awareness and enable them able to intervene when providing care for survivors" (P1) (P4).

The participants also noted that nowadays there are more community resources available. They can refer survivor to community organization, namely shelters and nongovernmental organizations and work with them to provide a more comprehensive response (P1). They also note that some networks have developed, and they are working more closely with communities and organizations (P4) (P5).

Another main issue with health providers is the systematic screening for intimate partner violence. Some trials have been made to include intimate partner violence in the screening process. Some local facilities will include the intimate partner violence screening in emergency departments. "We have included the screening for intimate partner violence in our Manchester screening protocol" (P2). On the other hand, another participant states that "some forms for screening intimate partner violence were created, but it depends on the will of decision makers" (P4).

\subsubsection{Impacts of Those Changes}

The participants had some further insights regarding several positive and negative impacts of the changes in the healthcare system.

1) Negative Impacts

The participants highlight that change in legislation that turned it into mandatory report is not followed. Healthcare professionals might make use of it but in most of the situations they face a dilemma of having to take care of the injuries but knowing that most survivors will have to go back and live with the perpetrator. The professionals report that they don't have the necessary resources to provide an ongoing support and doing risk assessment in order to protect the patients. The support depends on having a person available to network with other organizations. "They are more aware and recognize the signs but feel there is not much they can do" (P2) (P4).

Another concern is that with the cuts in funding some of the changes that were taking place were postponed and it will depend on the will of the decision makers (P4).

They also state that the changes in the health system are "very slow and there are many resistances from the medical professionals" (P5).

2) Positive

On the other hand the participants acknowledge an ever growing need of networking with other community stakeholders (P2). They are often called into the community to give an insight of the availability of the health system to be part of the resource network for the intervention in intimate partner violence (P4). A strong improvement has happened regarding the collaboration with police forces and other community organizations (P5). 


\subsubsection{Social Impacts}

All the participants stated that Portuguese society is more aware of intimate partner violence and less tolerant. Twenty years ago, women would have a hard journey to find support. Today, although women are more empowered a more profound social change is needed. Media used to blame the victim, nowadays perpetrators are held accountable. On the other hand, this issue has become so common that there is a risk of losing impact (P5). The professionals are more aware and available to listen and some organizations already have intervention protocols. In the healthcare system changes have been slow, but we already have many supportive and caring professionals (P1) (P3).

\subsubsection{Changes and Challenges for the Future}

Participants recognize the length of changes that have taken place, but they state that there is a lot to be done. They want in the future a more comprehensive and networked intervention. For that to happen, more training for professionals and more prevention programs are needed. Another issue is to reinforce the expertise of certain professionals in this area. Specialized teams should exist available around the clock so that people will not have to rely on chance to find a trained person.

\subsubsection{Intersections with Other Systems}

The healthcare system is only one of the actors involved in providing support for survivors of intimate partner violence. The participants in this study have clearly stated the need to work with professionals from other systems, namely the law enforcement system, the legal system and the community. The health system can benefit from the work done in other areas and is a nationwide resource available to provide emergency care.

\section{RESULTS}

Ideally, healthcare settings can provide a venue for enhancing intimate partner violence intervention but the efforts to reform the healthcare systems response to intimate partner violence proves to be difficult [9], and our study show us several examples of how difficult it has been.

Though the change process started in the early 1990's, it was since the year 2000 that it took a faster pace. Overall, our findings point to two major reforms that had a strong impact and fostered further changes in a system where changes are slow and with many resistances. Firstly, the changes in the law, that turned domestic violence into a public crime, and secondly, the change on the status of women in Portugal. The law has had a visible and clear impact in all stakeholders involved in intervention. Anyone can make a formal complain; meaning that the whole community is involved in preventing violence; it is a social problem that has to be everybody's concern. There are campaigns, funding for projects and training and an increase in the provision of community services. On the other hand, healthcare professionals will not approach the issue for fear of not having the skills or the resources to provide proper support and that survivors will have to go back home to a worse and increased risk situation. The change on the status of women in Portugal is a large scope issue that goes beyond intimate partner violence. But participants say that they have seen many changes on survivors-“they are more empowered", and there is a growing social intolerance to intimate partner violence and domestic abuse.

The current study further emphasizes the participant's view that training was not sufficient to foster the implementation of new practices systems-wide; the corresponding organizational changes and the will of decision makers are essential.

Thus, efforts to improve the health system response to domestic violence might consider other tactics that focus on creating changes in the organizational context by addressing issues at community level (e.g., working collaboratively with key stakeholders from the community, such as domestic violence advocacy organizations) [9].

Shepard states, referring to the USA that, "although we cannot point to one single intervention or set of interventions as the most significant, we can see that the multiple reforms over the past 2 decades have had some impact” (p. 437) [3]. This study shows that the Portuguese health system has made significant efforts to improve resources available for women survivors of violence, but in a non systematic way. Though the main changes acknowledged by our participants, the laws on domestic violence in 2000 and in 2009 and the changes over women's status over the past 40 years, are not directly related to the role of healthcare providers, it has had a strong impact on the health system response to intimate partner violence. Knowing that comprehensive institutional reforms can be successful in reducing intimate partner violence, and that changes in how social institutions reply to intimate partner violence reflect a shift in cultural sanctions for violence against women and have a ripple effect in the daily lives of women [3], our study shows that, though there has been significant change, we have yet to see a widespread and systematic transformation of the health system regarding intimate partner violence.

Since the examination of systems change is notoriously difficult due to the complexity and dynamic nature of systems [10], we should recognize the progress that has been made and continue our efforts to promote effecttive institutional practices. Anyhow, this study reveals the need for further studies on the health system in Portugal, namely, through the evaluation of procedures 
that were implemented over the last years and on the impacts of healthcare professional's response to survivors in preventing further violence.

\section{REFERENCES}

[1] Campbell, R., Patterson, D. and Cabral, G. (2010) Using ecological theory to evaluate the effectiveness of an indigenous community intervention: A study of sexual assault nurse examiner (SANE) programs. American Journal of Community Psychology, 46, 263-276. doi:10.1007/s10464-010-9339-4

[2] Goodman, L. and Smyth, K. (2011) A call for a social network-oriented approach for survivor of intimate partner violence. Psychology of Violence, 1, 79-92. doi:10.1037/a0022977

[3] Shepard, M. (2005) Twenty years of progress in addressing intimate partner violence: An agenda for the next 10. Journal of Interpersonal Violence, 20, 436-441. doi:10.1177/0886260504267879

[4] Allen, N., Bybee, D. and Sullivan, C. (2004) Battered women's multitude of needs. Violence against Women, 10, 1015-1035. doi:10.1177/1077801204267658

[5] Kreger, M., Brindis, C., Manuel, D. and Sassoubre, L. (2007) Lessons learned in systems change initiatives: Benchmarks and indicators. American Journal of Community Psychology, 39, 301-320. doi:10.1007/s10464-007-9108-1

[6] Sá Dias, I. (2000) Violência Doméstica em Portugal: Contributos para a sua Visibilidade. Actas do IV Congresso de Sociologia-Sociedade Portuguesa: Passados Recentes, Futuros Próximos. Consultado em 16 de Novembro de 2010.

http://www.aps.pt/Index.php?area=001\&marea=003\&id pub=PUB460a50b168fd1\&id_tema=TEM462dfc8fd6d91

[7] Usdin, S., Scheepers, E., Goldstein, S. and Japhet, G. (2005). Achieving social change on gender based violence: A report on the impact evaluation of soul city's fourth series.
Social Science \& Medicine, 61, 243-244. doi:10.1016/j.socscimed.2005.04.035

[8] WHO (2002) World report on violence and health. World Health Organization, Geneva.

[9] Allen, N., Lehrmer, A. and Mattisson, E. (2007) Promoting systems change in the healthcare response to intimate partner violence. Journal of Community Psychology, 35, 103-120.

[10] Javdani, S., Allen, N., Todd, N. and Anderson, C. (2010) Changes in the response to Intimate partner violence: Innovative aplications of multilevel modeling. Violence against Women, 17, 359-375.

[11] Kelly, J. (2006) Becoming ecological: An expedition into commmunity psycology. Oxford University Press, New York. doi:10.1093/acprof:oso/9780195173796.001.0001

[12] Foster-Fishman, P., Nowell, B. and Yang, H. (2007) Putting the system back into systems change: A framework for understanding and changing organizational and community systems. American Journal of Community Psychology, 39, 197-215. doi:10.1007/s10464-007-9109-0

[13] Behrens, T. and Foster-Fishman, P. (2007) Developing operating principles for systems change. American Journal of Community Psychology, 39, 411-414. doi:10.1007/s10464-007-9106-3

[14] Lehner, A. and Allen, N. (2009) Still a movement after all these years? Violence against Women, 15, 656-677. doi:10.1177/1077801209332185

[15] Bardin, L. (2006) Análise de Conteúdo. Lisboa, Edições.

[16] Lehner, A. and Allen, N. (2008) Social change movements and the struggle over meaning-making: A case study of domestic violence narratives. American Journal of Community Psychology, 42, 220-234.

[17] Barros, P.P., Lisboa, M., Cerejo, D. and Barrenho, E. (2008) Health care costs of domestic violence against women-Evidence from Portugal. doi:10.2139/ssrn.1160306 